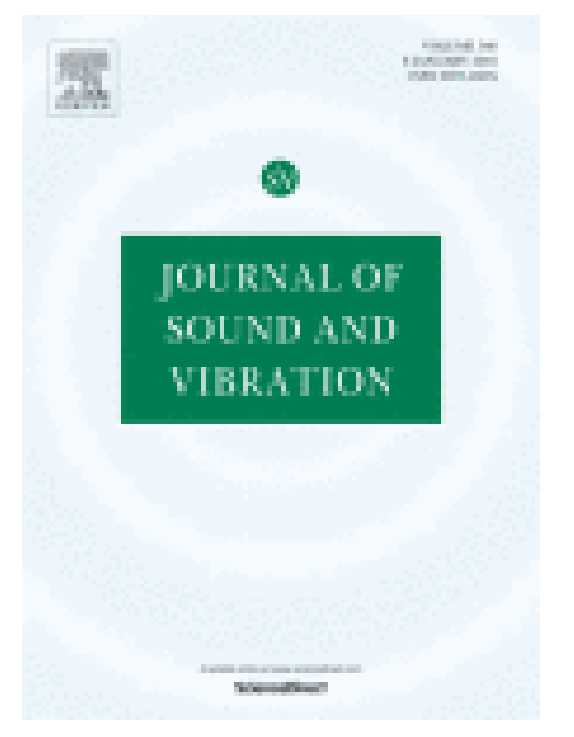

Title: "An analytical model of pneumatic suspensions based on an experimental characterization"

Authors: Nieto A.J., Morales A.L., Chicharro, J.M., Pintado $P$

JOURNAL OF SOUND AND VIBRATION, 313, pp. 290307 (2008)

Keywords:

Pneumatic suspension, stiffness, transmissibility

DOI: 10.1016/j.jsv.2007.11.027 


\title{
AN ANALYTICAL MODEL OF PNEUMATIC SUSPENSIONS BASED ON AN EXPERIMENTAL CHARACTERIZATION
}

\author{
A.J.NIETO, A.L.MORALES, A.GONZÁLEZ, J.M.CHICHARRO and P.PINTADO \\ Área de Ingeniería Mecánica \\ E.T.S.I.Industriales (Universidad de Castilla-La Mancha) \\ Avda Camilo José Cela s/n, 13071-Ciudad Real (Spain) \\ e-mail: antoniojavier.nieto@uclm.es
}

November 8, 2007

\begin{abstract}
We present an analytical model of air spring suspensions that is based on an experimental characterization. The suspension consists of three principal parts: the air spring, an auxiliary tank, and a pipe connecting the two. An analytical nonlinear fluid dynamics model is first analyzed, modeling the suspension stiffness, damping factor, and transmissibility. The model is then linearized and this linear version is studied in depth, finding that the behaviour of the suspension as reflected in the aforementioned three characteristics is strongly dependent on the size of the three suspension parts. The analysis allows us to propose a practical strategy for the operation of the suspension.
\end{abstract}

Keywords: Pneumatic suspension, stiffness, transmissibility, attenuation strategy.

PACS codes: 47.15.K-, 91.55.Ax, 47.27.nf 


\section{NOMENCLATURE}

\begin{tabular}{|c|c|}
\hline$A_{s}$ & Spring effective area $\left[\mathrm{m}^{2}\right]$ \\
\hline$C_{r}$ & The pipe restriction coefficient $\left[\mathrm{N}^{5} / \mathrm{m} / \mathrm{s}\right]$ \\
\hline$D_{p}$ & The pipe's cross section diameter $[\mathrm{m}]$ \\
\hline$F$ & Force exerted at the air spring $[\mathrm{N}]$ \\
\hline$g$ & Acceleration due to gravity $\left[\mathrm{m} / \mathrm{s}^{2}\right]$ \\
\hline$k$ & Pneumatic suspension total stiffness $[\mathrm{N} / \mathrm{m}]$ \\
\hline$k_{a s}$ & Air spring effective area stiffness $[\mathrm{N} / \mathrm{m}]$ \\
\hline$k_{s}$ & Air spring stiffness $[\mathrm{N} / \mathrm{m}]$ \\
\hline$k_{v s}$ & Air spring volume stiffness $[\mathrm{N} / \mathrm{m}]$ \\
\hline$k_{v s r}$ & Pneumatic suspension volume stiffness $[\mathrm{N} / \mathrm{m}]$ \\
\hline$l_{p}$ & The pipe length $[\mathrm{m}]$ \\
\hline M & Sprung mass $[\mathrm{kg}]$ \\
\hline$n$ & Polytropic coefficient \\
\hline$P_{r}$ & Relative pressure at the reservoir [bar] \\
\hline$P_{s}$ & Relative pressure at the air spring [bar] \\
\hline$R$ & Gas constant for air $[\mathrm{J} / \mathrm{kg} / \mathrm{K}]$ \\
\hline$T$ & Air suspension temperature $[\mathrm{K}]$ \\
\hline$V_{r}$ & Reservoir volume $\left[\mathrm{m}^{3}\right]$ \\
\hline$V_{s}$ & Air spring volume $\left[\mathrm{m}^{3}\right]$ \\
\hline$V_{s r}$ & Reservoir plus spring volume $\left[\mathrm{m}^{3}\right]$ \\
\hline$x$ & Absolute response $[\mathrm{m}]$ \\
\hline$y$ & Excitation $[\mathrm{m}]$ \\
\hline$z$ & Suspension height $[\mathrm{m}]$ \\
\hline$z_{0}$ & Initial height for the air spring $[\mathrm{m}]$ \\
\hline$\gamma$ & Specific heat ratio \\
\hline$\varepsilon$ & Dimensionless parameter \\
\hline$\theta$ & Dimensionless parameter \\
\hline$\mu$ & Dynamic viscosity of air $[\mathrm{Pa} \cdot \mathrm{s}]$ \\
\hline$\rho$ & Density of air $\left[\mathrm{kg} / \mathrm{m}^{3}\right]$ \\
\hline$\omega_{t r}$ & Transition frequency $[\mathrm{rad} / \mathrm{s}]$ \\
\hline$\dot{m}$ & Mass flow rate $[\mathrm{kg} / \mathrm{s}]$ \\
\hline
\end{tabular}




\section{Introduction}

The development of machinery and vehicles has increased the need for mechanical elements that are able to eliminate undesired vibrations. All vehicle suspensions are designed with the same end, i.e., to filter out vibrations coming from the tyre in contact with the road. One such suspension, the air suspension, has certain advantages over the classical mechanical suspensions in minimizing road wear and increasing passenger comfort. It is well known that air suspensions can provide both a soft ride at low speed on good roads and stability and control on rough roads at high speed. Moreover, the vehicle's chassis height can be adjusted to suit the particular conditions of any given trip [1].

The usefulness of pneumatic systems was demonstrated in [2], which addressed the problem of vibrations caused by vehicle cabins and their effect on the human body. The study showed that the need to add isolation to the vehicle cabins in order to prevent harmful vibrations from reaching the passengers was better served by seats with air suspension which attenuates frequencies above $4 \mathrm{~Hz}$ better than traditional suspension systems. Other studies have found benefits in air suspensions for seats in agricultural machinery ([3] and [4]) and in pneumatic systems in vibratory hand tools $([5])$.

The use of a resistance (discharge orifice) between an air spring and a chamber as a dissipation element to increase damping has been considered in several studies (an example is [6]). Although its practicality has been questioned ([7]) because the level of damping is amplitude dependent and unsuited to linear modeling [8], this damping element has been used to simulate with nonlinear techniques the behaviour of an ambulance stretcher fitted with a rigid wall linear cylinder air suspension [9], and to separate two chambers in a vibration isolator [10]. Another commonly used method of flow resistance is to include a valve with two parallel plates which restrict the air flow through the air suspension [1]. Nevertheless, these devices may be too cumbersome when there is need to implement a control strategy that modifies the suspension's characteristics.

There are several ways to incorporate said air spring suspensions. For example, in works like [11], a rigid wall pneumatic linear actuator is presented in parallel with an helical spring. The present work describes an air spring suspension with different pipes selected as the dissipation element together with an auxiliary volume. We study the system theoretically with both non- 
linear and linear models, and show how the linear model naturally suggests a specific control strategy.

\section{Nonlinear Model}

The pneumatic suspension we considered consists of three principal parts. The first is an air spring, the second component is a rigid tank and the third is a pipe connecting the first two elements. Consider first a closed system that includes only the air spring. The force exerted by the air spring can be written

$$
F=P_{s} A_{s}
$$

i.e., that the force is proportional to the air spring internal pressure, $P_{s}$, and the air spring effective area, $A_{s}$. The air spring stiffness can be defined as the derivative of this force with respect to the air spring height $z$ (defined as the difference between the response $x$ and the excitation $y$ plus an initial height value $z_{0}$ ):

$$
k_{s}=-\frac{d F}{d z}=-\left[A_{s} \frac{d P_{s}}{d z}+P_{s} \frac{d A_{s}}{d z}\right]
$$

A polytropic transformation, $P_{s} V_{s}^{n}=$ const, is assumed between an initial state (denoted by the additional subscript 0) and a final state. The derivative of air spring pressure with respect to height is then

$$
\frac{d P_{s}}{d z}=-\frac{n P_{s 0} V_{s 0}^{n}}{V_{s}^{n+1}} \frac{d V_{s}}{d z}
$$

where $P_{s 0}$ and $V_{s 0}$ are the air spring initial pressure and volume, respectively. The air spring stiffness can now be written as:

$$
k_{s}=\frac{n P_{s 0} V_{s 0}^{n} A_{s}}{V_{s}^{n+1}} \frac{d V_{s}}{d z}-P_{s} \frac{d A_{s}}{d z}
$$

By defining $k_{a s}$ and $k_{v s}$ as

$$
\begin{gathered}
k_{a s}=-P_{s} \frac{d A_{s}}{d z} \\
k_{v s}=\frac{n P_{s 0} V_{s 0}^{n} A_{s}}{V_{s}^{n+1}} \frac{d V_{s}}{d z}
\end{gathered}
$$

the air spring stiffness can be written as the sum of two terms: a stiffness term, $k_{a s}$, due to the air spring effective area variation with height $z$, and a volume term, $k_{v s}$, which depends on the air spring volume variation with height $z$. 
As was mentioned above, the stiffness $k_{s}$ corresponds to a closed system consisting of the air spring alone, i.e., the auxiliary reservoir has not as yet been taken into account in the model. Nevertheless, the pressures in the air spring and the reservoir can be assumed to be the same at any given instant (provided that the pipe connecting the two chambers is open) if the suspension dynamics are sufficiently slow. If this assumption holds, one can use Eq. (4) to obtain the suspension stiffness, $k$, by adding the tank volume $\left(V_{r}\right)$ to that of the spring:

$$
k=\frac{n P_{s 0} V_{s r 0}^{n} A_{s}}{V_{s r}^{n+1}} \frac{d V_{s}}{d z}-P_{s} \frac{d A_{s}}{d z}
$$

where $V_{s r}$ is the sum of the volume of the air spring, $V_{s}$, and the tank volume, $V_{r}$ and, thus, $V_{s r 0}$ is the initial sum of volumes. In this case, the pneumatic suspension stiffness is $k=k_{a s}+k_{v s r}$, and the stiffness term due to the suspension volume variation with respect to height is now:

$$
k_{v s r}=\frac{n P_{s 0} V_{s r 0}^{n} A_{s}}{V_{s r}^{n+1}} \frac{d V_{s}}{d z}
$$

The functions $A_{s}(z)$ and $V_{s}(z)$ and their derivatives terms, $d A_{s} / d z$ and $d V_{s} / d z$, will be determined experimentally as described in the following section.

At the other end of the spectrum, when the suspension dynamics is very fast, the pressure waves do not have time to reach the reservoir. Therefore, at high frequencies, the suspension behaves like a closed system formed by the air spring alone. The stiffness in this case is given by Eq. (2).

We modeled the stiffness for these two extreme scenarios: very slow dynamics (low frequency range) in which case the stiffness is given by Eq. (7), and very fast dynamics (high frequency range) in which case the stiffness is given by Eq. (2). In the following, we will describe a fluid dynamics model for the general case. The mass flow from the air spring to the reservoir can be expressed by the continuity equation:

$$
\dot{m}=-\dot{\rho}_{s} V_{s}-\dot{V}_{s} \rho_{s}
$$

the flow being positive when filling the bellows.

The suspension air temperature was monitored experimentally in working conditions by means of a thermocouple. The results supported the hypothesis of an isothermal transformation. With this hypothesis, the air spring density variation with respect to time can be defined as follows:

$$
\dot{\rho}_{s}=\frac{\dot{P}_{s}}{R T}
$$


where the ideal gas equation, $P_{s}=\rho_{s} R T$, has been assumed. Combining Eqs. (9) and (10) yields:

$$
\dot{P}_{s}=-\dot{m} \frac{R T}{V_{s}}-\dot{V}_{s} \frac{P_{s}}{V_{s}}
$$

The same steps followed to obtain Eq. (11) are now used at the reservoir end in order to link the mass flow rate to the reservoir pressure $\left(\dot{V}_{r}\right.$ is equal to 0 because the walls of the reservoir are assumed rigid):

$$
\dot{P}_{r}=\dot{m} \frac{R T}{V_{r}}
$$

where $P_{r}$ is the auxiliary reservoir pressure. The rate of change of reservoir pressure connected to a pipe is the same rate one would have in a discharge process [12], and may be written as

$$
\dot{P}_{r}=-\frac{\gamma C_{r}}{2 V_{r}}\left(P_{r}^{2}-P_{s}^{2}\right)
$$

where $\gamma$ is the specific heat ratio and $C_{r}$ the restriction coefficient defined as [13]:

$$
C_{r}=\frac{\pi D_{p}^{4}}{128 \mu l_{p}}
$$

where $l_{p}$ is the pipe length, $D_{p}$ is the pipe's cross section diameter, and $\mu$ the dynamic viscosity of air. Previous work on the type of flow (taking into account the size of the pipes and the amplitude and frequency of the experimental excitation signal) allow us to consider an incompressiblefully-developed-laminar flow (Hagen-Poiseuille flow), in particular, Mach number $M<0.3$ and Reynolds number $R e<2300$. Eqs. (11) and (12) are combined to yield:

$$
\dot{P}_{s}=-\dot{P}_{r} \frac{V_{r}}{V_{s}}-\dot{V}_{s} \frac{P_{s}}{V_{s}}
$$

Two additional equations are now incorporated to complete the analytical model. One is the variation of the air spring force with respect to time, and can be obtained from the derivative of Eq. (1):

$$
\dot{F}=\dot{P}_{s} A_{s}+P_{s} \dot{A}_{s}
$$

The other is Newton's second law:

$$
M \ddot{x}+M g-F=0
$$

with $M$ being the sprung mass, $x$ its dynamic response, and $g$ the acceleration due to gravity. The variations of the air spring effective area and volume with respect to time $\left(\dot{A}_{s}\right.$ and $\left.\dot{V}_{s}\right)$ that appear in Eqs. (15) and (16) can be written as:

$$
\dot{A}_{s}=\frac{d A_{s}}{d z} \frac{d z}{d t}=A_{s}^{\prime}(\dot{x}-\dot{y})
$$




$$
\dot{V}_{s}=\frac{d V_{s}}{d z} \frac{d z}{d t}=V_{s}^{\prime}(\dot{x}-\dot{y})
$$

Eq. (15) is combined with Eq. (19) to obtain the first equation in the system below. The second equation of this system is equal to Eq. (13). The third equation in the system is obtained combining the derivative with respect to time of Eq. (17) with Eqs. (16) and (18). With all these expressions put into order, one finally has the following system of differential equations:

$$
\left\{\begin{array}{l}
\dot{P}_{s}=-\dot{P}_{r} \frac{V_{r}}{V_{s}}-V_{s}^{\prime} \frac{P_{s}}{V_{s}}(\dot{x}-\dot{y}) \\
\dot{P}_{r}=-\frac{\gamma C_{r}}{2 V_{r}}\left(P_{r}^{2}-P_{s}^{2}\right) \\
M \dddot{x}=\dot{P}_{r} A_{s}+P_{s} A_{s}^{\prime}(\dot{x}-\dot{y})
\end{array}\right.
$$

This system has only three unknown functions of time, $P_{s}, P_{r}$, and $x$. The other elements are known parameters $\left(M, V_{r}, C_{r}\right.$, and $\left.y\right)$ or functions that have to be determined experimentally $\left(A_{s}(z)\right.$ and $\left.V_{s}(z)\right)$.

\subsection{Experimental Characterization of the Air Spring}

The previously presented model depends on two functions of height that need to be determined experimentally. These functions are the air spring effective area and the air spring volume. Two trials were performed in order to characterize the suspension. The set-up included neither the tank nor the connecting pipe.

First, to determine the air spring effective area, the spring was locked at its upper end, and its lower end, fixed by the hydraulic gag, was subjected to the displacement excitations. The test input motion was a monotonic displacement in which the height $z$ changes from $0.09 \mathrm{~m}$. to $0.175 \mathrm{~m}$. The air spring pressure was kept constant by means of a pressure control valve. This trial was performed for three constant pressures: 2,3 , and 4 bar. The effective area is the ratio between the exerted force as measured from the hydraulic actuator load cell, and the constant pressure at which the test was carried out.

Then, to determine the variation of the air spring volume with height, a pressure controlled pump was used to fill the spring with water keeping the pressure constant. The height $z$ was again varied as in the previous trial from $0.09 \mathrm{~m}$ to $0.175 \mathrm{~m}$. This means that the pressure controlled pump had to fill or empty the spring, depending on that height, to maintain its 
pressure at a constant value. This procedure was carried out for several values of the air spring height. For each height, the water inside the air spring was retrieved and weighed to measure the volume at that particular height. The tests were performed for different pressures (the same as were used for the effective area case).

Fig. 1a shows the effective area versus height for the three pressures, and Fig. 1b volume versus height for the same three pressures. As can be seen both area and volume are almost independent of pressure, and, importantly, the two functions are fairly close to linear.

\subsection{Numerical Solution}

The system of differential equations (20) were solved numerically by substituting the experimental characterization described in the previous section. The processing was done in the Matlab Simulink environment [14].

The principal block diagram for the model is shown in Fig. 2. The model input function is the excitation $y$ and the output functions are the air spring and reservoir pressures $\left(P_{s}\right.$ and $\left.P_{r}\right)$ and the dynamic response $(x)$. This main scheme is divided into three secondary blocks representing the three differential equations of system (20). These three secondary blocks yield the unknown functions $x, P_{s}$, and $P_{r}$, which are then re-introduced to their own and the other blocks in a process of iteration, as shown in Fig. 2. Besides these functions, the other experimentally obtained constants or functions of the height are substituted into each particular block.

The pneumatic suspension's stiffness is first simulated. Four different types of pipes represented by their $C_{r}$ coefficients are used. A frequency range from 0.1 to $25 \mathrm{~Hz}$ was used in the analysis. The results of this simulation can be seen in Fig. 3. All curves show relatively constant stiffness zones at low and high frequencies and an intermediate transition zone connecting them. This stiffness behavior pattern is repeated for every $C_{r}$ coefficient. Nevertheless, the transition frequencies grow with $C_{r}$. The suspension's Carding diagram is plotted in Fig. 4 for every type of pipe. These diagrams are obtained by simulating using a stroke of $\pm 5 \mathrm{~mm}$ above and below the air spring initial height $z_{0}$. Four different diagrams (for four different excitation frequencies) are presented for each $C_{r}$ coefficient. 
The Carding diagrams show the variation of stiffness (overall slope of the loop) and of damping (area inside the loop) with frequency. Damping grows with frequency up to a maximum value from which damping decreases as frequency grows. The corresponding transition frequency grows with $C_{r}$. The frequency range where damping grows can also be observed in the Bode plot phase diagrams of Fig. 3. One sees that, at low and high frequencies, the signals $F(s)$ and $Z(s)$ are in phase opposition. At intermediate frequencies, there is a time delay between $F(s)$ and $Z(s)$ for all the stiffness curves, with roughly the same phase curve shape being repeated at earlier or later frequencies depending on the value of $C_{r}$. The greater the $C_{r}$ pipe coefficient, the higher the frequency needed to reach the high frequency limit.

The suspension response to step displacement inputs is now analyzed for the two extreme $C_{r}$ values. Two different step displacement sizes have been used: $10 \mathrm{~mm}$ and $2.5 \mathrm{~mm}$. Results are shown in Fig. 5. It is easy to notice that the suspension configuration for the lower $C_{r}$ coefficient shows a higher stiffness, and therefore a higher natural frequency, than the system with higher $C_{r}$ coefficient.

The pneumatic suspension's transmissibility may finally be obtained. The results have been focused on the frequency range from 0.5 to $7 \mathrm{~Hz}$. This simulation is shown in Fig. 6. The $C_{r}$ influence on the suspension eigenfrequencies (already seen in Fig. 5) is again detected.

\section{Linearized Model}

In this section, we will present a first-order Taylor series expansion of the nonlinear differential equations of Sec. 2. This Taylor series is obtained in the neighbourhood of the static equilibrium point denoted by superscript $s t$ and defined by the following values:

$$
\begin{aligned}
& \dot{m}^{s t}=0 ; \quad A_{s}=A_{s}^{s t} ; \quad z^{s t}=z_{0} ; \\
& P_{s}=P_{r}=P_{r}^{s t}=P_{s}^{s t} ; \quad \dot{P}_{s}^{s t}=0 ; \quad \dot{P}_{r}^{s t}=0 ; \\
& A_{s}^{\prime s t}=\lambda ; \quad V_{s}=V_{s}^{s t} ; \quad V_{s}^{\prime s t}=\kappa ; \quad \dot{F}^{s t}=0 ; \quad \dot{z}^{s t}=(\dot{x}-\dot{y})^{s t}=0 ;
\end{aligned}
$$

The linearized version of the system of differential equations is the following:

$$
\dot{P}_{s}=-\dot{P}_{r} \frac{V_{r}}{V_{s}^{s t}}-\kappa \frac{P_{s}^{s t}}{V_{s}^{s t}}(\dot{x}-\dot{y})
$$




$$
\begin{gathered}
\dot{P}_{r}=-\frac{\gamma C_{r} P_{s}^{s t}}{V_{r}}\left(P_{r}-P_{s}\right) \\
M \dddot{x}=A_{s}^{s t} \dot{P}_{s}-\lambda P_{s}^{s t}(\dot{x}-\dot{y})
\end{gathered}
$$

Applying Laplace's transform to Eqs. (21), (22) and (23), one obtains the stiffness transfer function as follows:

$$
\frac{F(s)}{Z(s)}=-\frac{s\left(K_{A S}+K_{V S}\right)+W K_{V S}\left(1+\frac{K_{A S}}{K_{V S R}}\right)}{s+W\left(\frac{K_{V S}}{K_{V S R}}\right)}
$$

where $F(s)$ is the Laplace transform of $F(t), Z(s)$ is the Laplace transform of $z(t)=x(t)-$ $y(t)+z_{0}, W$ is defined as $W=\gamma C_{r} P_{s}^{s t} / V_{r}$, and the terms $K_{A S}, K_{V S}$, and $K_{V S R}$ are defined as:

$$
\begin{gathered}
K_{A S}=-P_{s}^{s t} \lambda=\left.k_{a s}\right|^{s t} \\
K_{V S}=\frac{P_{s}^{s t} A_{s}^{s t}}{V_{s}^{s t}} \kappa=\left.k_{v s}\right|^{s t} \\
K_{V S R}=\frac{P_{s}^{s t} A_{s}^{s t}}{V_{s}^{s t}+V_{r}} \kappa=\left.k_{v s r}\right|^{s t}
\end{gathered}
$$

and therefore the spring stiffness and suspension stiffness defined in Eqs. (4) and (7) are now written as

$$
\begin{aligned}
& K_{S}=k_{s}^{s t}=K_{A S}+K_{V S} \\
& K=k^{s t}=K_{A S}+K_{V S R}
\end{aligned}
$$

The linearized Eq. (21), (22), and (23) written in the Laplace domain are also used to obtain the transmissibility transfer function. This function is the ratio between the response $X(s)$ and the excitation $Y(s)$ :

$$
\frac{X(s)}{Y(s)}=\frac{s\left(\frac{K_{A S}+K_{V S}}{M}\right)+W \frac{K_{V S}}{M}\left(1+\frac{K_{A S}}{K_{V S R}}\right)}{s^{3}+s^{2}\left(W \frac{K_{V S}}{K_{V S R}}\right)+s\left(\frac{K_{A S}+K_{V S}}{M}\right)+W \frac{K_{V S}}{M}\left(1+\frac{K_{A S}}{K_{V S R}}\right)}
$$

It is convenient to write this last equation in a dimensionless form. For this purpose, one defines the dimensionless parameters $\theta$ and $\varepsilon$ :

$$
\begin{gathered}
\theta=\sqrt{\frac{K_{A S}+K_{V S}}{K_{A S}+K_{V S R}}} \\
\varepsilon=\frac{W}{\omega_{s} \theta^{2}} \frac{K_{V S}}{K_{V S R}}
\end{gathered}
$$

where $\omega_{s}=\sqrt{K_{S} / M}$. These two parameters are inserted into Eq. (30) yielding the dimensionless transmissibility transfer function:

$$
\frac{X(s)}{Y(s)}=\frac{s\left(\frac{1}{\omega_{s} \varepsilon}\right)+1}{s^{3}\left(\frac{1}{\omega_{s}^{3} \varepsilon}\right)+s^{2}\left(\frac{\theta^{2}}{w_{s}^{2}}\right)+s\left(\frac{1}{\omega_{s} \varepsilon}\right)+1}
$$


These expressions will be used next to obtain some conclusions about the behavior of the pneumatic suspension. In Fig. 3 of previous section, the suspension stiffness was simulated by the nonlinear model. It was observed that the stiffness may be divided into two zones corresponding to low and high frequencies. All the stiffness curves for the four different values of $C_{r}$ rise from a lower limiting value at low frequencies to an upper limiting value at high frequencies. These two limiting values do not depend on $C_{r}$, and correspond to those obtained with the simplified models of Sec. 2. In particular, the low frequency stiffness coincides with that of Eq. (7), and the high frequency stiffness with that of Eq. (4). These limiting values of stiffness of those two equations can also be obtained from the linear model. At low frequencies, the Laplace transform of the stiffness tends to

$$
\left.\frac{F(s)}{Z(s)}\right|_{s \Rightarrow 0}=K_{A S}+K_{V S R}
$$

which gives a value of stiffness equal to $K$. At high frequencies, the Laplace transform of the stiffness tends to

$$
\left.\frac{F(s)}{Z(s)}\right|_{s \Rightarrow \infty}=K_{A S}+K_{V S}
$$

which gives a value of stiffness equal to $K_{S}$. The transitions between these two values take place at frequencies that depend on the coefficient $C_{r}$.

In Fig. 6, the eigenfrequency corresponding to the largest $C_{r}$ value is close to the lowest achievable. Indeed, this eigenfrequency may be calculated approximately by assuming the pneumatic suspension stiffness in this situation to be the linear model stiffness at low frequencies, $K$. Similarly, the eigenfrequency corresponding to the smallest $C_{r}$ coefficient is close to the highest achievable, and can be calculated approximately by assuming the stiffness in this situation to be $K_{S}$. Consider now the dimensionless parameter $\varepsilon$ related to the pipe restriction coefficient that were defined above. The two limits as $\varepsilon$ tends to zero and to infinity yields:

$$
\begin{gathered}
\left.\frac{X(s)}{Y(s)}\right|_{\varepsilon \Rightarrow 0}=\frac{1}{s^{2} \frac{1}{\omega_{s}^{2}}+1} \\
\left.\frac{X(s)}{Y(s)}\right|_{\varepsilon \Rightarrow \infty}=\frac{1}{s^{2} \frac{\theta^{2}}{\omega_{s}^{2}}+1}=\frac{1}{s^{2} \frac{1}{\omega^{2}}+1}
\end{gathered}
$$

defining $\omega$ as $\sqrt{K / M}$. This limits make it possible to understand the meaning of the kind of pipe connection between the reservoir and the air spring. The limit as $\varepsilon$ tends to zero corresponds to a small coefficient $C_{r}$, and the Eq. (36) is that of a typical non-damped system with an equivalent stiffness $K_{S}$, and it can be seen that the system behaves as an air spring suspension with no 
contribution from the reservoir. The limit as $\varepsilon$ tends to infinity, Eq. (37), means that, for large values of the coefficient $C_{r}$, the behaviour is that of a non-damped system with an equivalent stiffness $K$, corresponding to the suspension working as the air spring with an increased volume coming from the reservoir.

\section{Experimental Results}

The suspension stiffness, damping, and transmissibility are now measured with an experimental air spring suspension for comparison with our previous development of this system model. The pneumatic suspension we considered consists of three principal parts as it was said before. The first is the air spring itself (M/31062 of Norgren). This is a two-bellow air spring, with 90 $\mathrm{mm}$ total stroke, made of reinforced rubber (SBR), and functional from $-40^{\circ} \mathrm{C}$ to $70^{\circ} \mathrm{C}$ and up to 8 bar. The second component is a $2 \mathrm{l}$ capacity steel tank. And the third is a nylon pipe connecting the first two elements. The dimensions of this pipe were changed in several ways (according to the ones whose $C_{r}$ coefficient was selected in the models) for their influence to be studied. Indeed, the sizes of the pipe, tank, and air spring were found to be the most important design parameters in determining the suspension's behaviour.

Fig. 7 shows the experimental set-up. Two types of trials were performed on a displacement controlled hydraulic actuator (MTS 810): the pneumatic suspension stiffness was measured with the set-up shown in Fig. 7a, and the suspension damping and the sprung mass absolute response, i.e., the system's transmissibility, was measured with the set-up shown in Fig. 7b. For the stiffness measurements, the top of the air spring is locked by the hydraulic actuator's upper gag, and the bottom gag exerts the excitation. For the transmissibility measurements, the upper gag holds a linear bearing that guides the sprung mass placed on top of the pneumatic suspension, and again the suspension excitation comes from the bottom gag.

The pipe sizes were selected with widths in the range 2.7 to $8 \mathrm{~mm}$, and lengths of 0.5 to 2 $\mathrm{m}$. The initial air spring height was set at $130 \mathrm{~mm}$. The air spring initial internal pressure was 3 bar for the stiffness measurements, and 1 bar for the transmissibility measurements, the latter internal pressure chosen to equilibrate the sprung mass static load $(120 \mathrm{~kg})$. With a higher initial pressure, a larger mass must be mounted. The sprung mass in the present trials was chosen as a 
function of the work space allowed by the hydraulic unit. The total air mass (including bellows, pipe, and tank) was held constant.

The displacement input signal for the stiffness trials was a $5 \mathrm{~mm}$ amplitude sine wave applied by the hydraulic actuator. The frequency range tested was 0.1 to $25 \mathrm{~Hz}$. The output signal was the force wave as measured by the hydraulic actuator load sensor. The results will be presented for four different pipes characterized by their corresponding restriction coefficient $C_{r}$ (the same that in models). Fig. 8 shows the suspension stiffness obtained using the nonlinear model, the linear model and the experimental results. The stiffness is shown both in magnitude (Figs. 8a, $8 \mathrm{c}, 8 \mathrm{e}$ and $8 \mathrm{~g}$ ) and phase (Figs. 8b, 8d, $8 \mathrm{f}$ and $8 \mathrm{~h}$ ) for four different values of $C_{r}$. As can be seen in the figure, the behavior of the suspension at the workbench has been well predicted both by the nonlinear and the linear models. The experimentally obtained Carding diagrams are shown in Fig. 9. The diagrams are well in agreement with those of Fig. 4.

The test set-up shown in Fig. 7b was used to measure the suspension damping (by means of the step response test). The hydraulic actuator provides a square signal that can be used as a step input. The frequency of that square signal was chosen to be slow enough for the attenuated output signal (sprung mass displacement) to be acquired properly. As in the nonlinear model simulation, two step displacement sizes have been used in the experimental case: 10 and 2.5 mm. The results of this test can be seen in Fig. 10.

Fig. 11 shows the experimental and the linear model normalized step responses of the suspension for the smallest and the largest values of $C_{r}$ (Figs. 11a and 11b respectively). Table 1 presents the values of the suspension damping factor and eigenfrequencies calculated with the linear model for these two $C_{r}$ values, together with the experimental values. The experimental dimensionless damping factor $\xi$ was estimated by means of the logarithmic decrement technique [15], giving values for these damping factors of from 0.119 to 0.085 , where the lower values corresponding to pipes with larger and smaller $C_{r}$ coefficients. One observes that the linear model was able to predict the suspension behaviour quite well with respect to its stiffness, and hence its eigenfrequencies. The damping factor prediction was less accurate, however, although it is commonly found to be difficult to construct a good model to predict the damping. Indeed, in many cases this damping factor is determined experimentally.

Finally, the same set of four pipes were used for the transmissibility test, but now with a 1.5 
$\mathrm{mm}$ sine wave input signal and a frequency range from 0.5 to $7 \mathrm{~Hz}$. The displacement output signal was captured by an LVDT (Schaevitz DC-EC 2000) with $\pm 50 \mathrm{~mm}$ measurement range and $0.01 \mathrm{~mm}$ resolution assembled onto the sprung mass. Fig. 12 shows the transmissibility using the nonlinear model, the linear model and the experimental tests. This figure confirms that both models are in good approximation to the measured data.

\section{Operation strategy}

The modeling and testing of a pneumatic suspension have been useful in attaining a better understanding of its behaviour, including the prediction of its dynamic response. However, as was seen, this type of suspension has many modes of operation depending on the choice of elements. One therefore requires an operational strategy to ensure getting the optimal performance of a suspension. Some examples of such strategies are presented in [9] and [16]. The former suggests using the well-known sky-hook principle and changing the size of an orifice, used as the air flow restriction element, according to the sign of the difference between the air spring and volume pressures. The latter suggests using a switchable viscous damper in series with a relaxation spring. Neither suggestion is straightforward to apply in practice. Another idea for suspensions of this type was put forward in [17]. The aim was to achieve a suspension whose dynamic response, as reflected in its transmissibility curves, has a special characteristic. Specifically, they look for the transmissibility curve with the lowest maximum, i.e., the strategy is to look for the best attenuation of the excitation. The idea is only practical, however, over a narrow frequency range. A meaningful improvement suggests itself if one observes the linear model transmissibility diagram (Fig. 13) in detail. In the magnitude diagram of that figure (Fig. 13a) one sees that there is a point through which all the curves pass. The difference between these curves, as was seen above, is related to the coefficient $C_{r}$, and therefore to the dimensionless parameter $\varepsilon$. The point can be calculated analytically in the model by assuming the following condition:

$$
\frac{\partial\left|\frac{X}{Y}\right|}{\partial \varepsilon}=0
$$

The frequency corresponding to that point is called the transition frequency $\left(\omega_{t r}\right)$, and is obtained as:

$$
\omega_{t r}=\omega_{s} \sqrt{\frac{2}{\theta^{2}+1}}
$$


while the value of the transmissibility modulus at this transition frequency is

$$
\left|\frac{X}{Y}\right|_{t r}=\frac{\theta^{2}+1}{\theta^{2}-1}
$$

where $\theta$ is the dimensionless parameter defined in Eq. (31).

The identification of this transition point allows one to establish an operational strategy. On each side (left and right) of this point, there is a transmissibility curve whose amplification is the smallest least. These two curves do not, however, correspond to the same value of $C_{r}$. In other words, to get these results, one needs to use two different pipes. This may not be a problem if the transition point is used as a switching boundary. One would then need two pipes for this strategy: one with the smallest and one with the largest value of the coefficient $C_{r}$. The transition point indicates where a change must be made from using one pipe to using the other. A switching valve would have to be used for this task, selecting the smaller $C_{r}$ coefficient pipe if the excitation frequency was less than $\omega_{t r}$, and the larger one otherwise.

Once the frequency $\omega_{t r}$ has been determined, the following task is to improve the features of the suspension, i.e., to achieve the smallest possible value of the transmissibility modulus (corresponding to that frequency $\omega_{t r}$ ). The modulus, as Eq. (40) shows, is a function of the dimensionless parameter $\theta$. Since the greater the value of this parameter, the smaller the modulus, the selection of the suspension elements must be aimed at increasing the value of $\theta$ as much as possible. In other words, one must seek the greatest distance possible between the eigenfrequency values corresponding to the smallest and highest $C_{r}$ coefficients in the transmissibility diagram by maximizing and minimizing the values of $K_{V S}$ and $K_{V S R}$, respectively. This involves reducing the air spring volume $V_{s}$ and increasing the reservoir volume $V_{r}$.

Reducing the air spring volume along with increasing the reservoir make it possible (as it has been argued above) to reduce the transmissibility modulus at transition frequency $\omega_{t r}$. At the same time, this modulus reduction may improve the results of the control strategy when the aim is to filter single-frequency signals. The active control method just explained may also be useful when filtering vibrations caused by unbalanced rotating machines subjected to frequent start-stop cycles. Nevertheless, this active control strategy may be used as a basis in order to attenuate random vibrations. The details of the process are beyond the scope of this work, but it can be said that it will involve estimation of the excitation power spectral density (PSD), as 
well as criteria to switch between pipes of different sizes. To this end, real-time PSD estimation techniques (as proposed in [18]) will be needed.

\section{Conclusions}

We have presented an analytical model of the behavior of an air spring based on an experimental characterization. The solution of both the nonlinear model and its linearized version are well in agreement with our experimental measurements of the stiffness, damping factor, and transmissibility for a reasonable operation range of this suspension. It was shown that the dynamic behavior of the air suspension can be made more versatile by a convenient choice of the sizes of the its elements, in particular of the air spring and reservoir volumes. On the one hand, reducing the air spring volume increases the stiffness and hence also the highest eigenfrequency. And on the other hand, increasing the reservoir volume reduces the stiffness and hence also the lowest eigenfrequency.

Therefore, by implementing these changes in the elements of the suspension one can increase the difference between these two eigenfrequencies, i.e., one can increase the $K_{S} / K$ ratio. The utility of this strategy would come into play if the air suspension were designed to work differently in two frequency regions. The working frequency range is naturally divided into two parts by a point at the "transition frequency" through which all the transmissibility curves pass. To achieve reasonable vibration attenuation, for low frequencies below this point one would choose the pipe with the smallest $C_{r}$ coefficient, and for high frequencies above this point the pipe with the largest coefficient. This would require a control system capable of deciding and choosing the correct pipe to use according to the excitation frequency. If the suspension elements are not changed, the stiffness values $K_{A S}, K_{V S}$, and $K_{V S R}$ are constant (if the initial working pressure is also constant). The value of the transition frequency, depends on the parameter $\theta$ which is a function of these three stiffness values. Furthermore, $\omega_{t r}$ depends on $\omega_{s}$, i.e., on the sprung mass, so that varying this mass changes the value of $\omega_{t r}$. Designing and implementing a control system that improves the suspension's behaviour according to the excitation frequency and the value of the initial sprung mass will be part of the authors' ongoing work. 


\section{Acknowledgement}

The authors are grateful for the support received from the Regional Project PBI05-020 entitled "Análisis y diseño de elementos neumáticos activos para el control de vibraciones" financed by the Consejería de Educación y Ciencia (Junta de Comunidades de Castilla-La Mancha).

\section{References}

[1] E.Esmailzadeh, "Optimization of pneumatic vibration isolation system for vehicle suspension", Journal of Mechanical Design, 100, (1978), 500-506.

[2] I.Hostens, H.Ramon, "Descriptive analysis of combine cabin vibration and their effect on the human body", Journal of Sound and Vibration, 266, (2003), 453-464.

[3] I.Hostens, K.Deprez, H.Ramon, "An improved design of air suspension for seats of mobile agricultural machines", Journal of Sound and Vibration, 276, (2004), 141-156.

[4] K.Deprez, D.Moshou, J.Anthonis, J.De Baerdemaeker, H.Ramon, "Improvement of vibrational comfort on agricultural vehicles by passive and semi-active cabin suspensions", Computers and Electronics in Agriculture, 49, (2005), 431-440.

[5] R.Palej, S.Piotrowski, M.Stojek, "Mechanical properties of an active pneumatic spring", Journal of Sound and Vibration, 168(2), (1993), 299-306.

[6] G.Quaglia, M.Sorli, "Experimental and theoretical analysis of an air spring with auxiliary reservoir", Proceedings of the 6th International Symposium on Fluid Control Measurement and Visualization, Sherbrooke, Canada, 2000.

[7] J.K.Baker, "Vibration Isolation", 1975, Engineering Design Guides 13 (Oxford University Press for the Design Council).

[8] S.Koyanagi, "The development of the air spring with variable nozzle", Quarterly Report of Railway Technical Research Institute, 31 (3), (1990), 122-127.

[9] R.J.Henderson, J.K.Raine, "A two-degree-of-freedom ambulance stretcher suspension", Proceeding Institution of Mechanical Engineers. 212, Part D, (1998), 227-240. 
[10] C.Erin, B.Wilson, "An improved model of a pneumatic vibration isolator: theory and experiment", Journal of Sound and Vibration, 218(1), (1998), 81-101.

[11] B.I.Bachrach, "Analysis of a damped pneumatic spring", Journal of Sound and Vibration, 86(2), (1983), 191-197.

[12] E.Fermi, "Thermodynamics", Dover Publications, 1996.

[13] I.H.Shames, "Mechanics of fluids", Second edition. McGraw-Hill, 1982.

[14] MathWorks, 1999. Matlab 6.1.1., Copyright (c) by the MathWorks Inc., Natick, MA.

[15] S.S.Rao, "Mechanical vibrations", Third edition. Addison-Wesley, 1995.

[16] C.W.Stamers, "The role of a relaxation damper in a semi-active suspension", In IMechE Autotech 1993, Birmingham, 1993, paper C462/32/014 (Mechanical Engineering Publications, London).

[17] J.I.Soliman, D.Tajer-Ardabili, "Self-damped pneumatic isolator for variable frequency excitation", Journal of Mechanical Engineering, 8(3), (1966), 284-293.

[18] K.Kanazawa, K.Hirata, "Parametric estimation of the cross-power spectral density", Journal of Sound and Vibration, 282, (2005), 1-35. 


\section{Figure captions}

Figure 1. Experimental characterization of (a) the air spring effective area, and (b) the air spring volume.

Figure 2. General Simulink block diagram for the nonlinear model of the pneumatic suspension.

Figure 3. Nonlinear simulation (stiffness modulus and phase diagrams) for the suspension frequency response: (a) and (b) for $C_{r} \approx 10^{-8}$; (c) and (d) for $C_{r} \approx 10^{-7}$; (e) and (f) for $C_{r} \approx 10^{-6}$; (g) and (h) for $C_{r} \approx 10^{-5}$.

Figure 4. Nonlinear simulation for the suspension Carding diagram: a) for $C_{r} \approx 10^{-8}$; (b) for $C_{r} \approx 10^{-7} ;(\mathrm{c})$ for $C_{r} \approx 10^{-6} ;$ (d) for $C_{r} \approx 10^{-5}$.

Figure 5. Nonlinear simulation for the suspension step response.

Figure 6. Nonlinear simulation for the suspension transmissibility: a) for $C_{r} \approx 10^{-8}$; (b) for $C_{r} \approx 10^{-7} ;$ (c) for $C_{r} \approx 10^{-6} ;(\mathrm{d})$ for $C_{r} \approx 10^{-5}$.

Figure 7. Experimental set-up: (a) stiffness measurements with no suspended mass; (b) damping and transmissibility measurements with suspended mass.

Figure 8. Comparison of the nonlinear model (thin solid lines) and linear model (thick solid lines) with experiment for the stiffness (modulus and phase diagrams) of the pneumatic suspension: (a) and (b) for $C_{r} \approx 10^{-8}$; (c) and (d) for $C_{r} \approx 10^{-7}$; (e) and (f) for $C_{r} \approx 10^{-6}$; (g) and (h) for $C_{r} \approx 10^{-5}$.

Figure 9. Test results for the suspension Carding diagram: a) for $C_{r} \approx 10^{-8}$; (b) for $C_{r} \approx 10^{-7}$; (c) for $C_{r} \approx 10^{-6}$; (d) for $C_{r} \approx 10^{-5}$.

Figure 10. Test results for the suspension step response.

Figure 11. Comparison of the linear model (solid lines) with experiment for the suspension step response of the pneumatic suspension: (a) for $C_{r} \approx 10^{-8}$; (b) for $C_{r} \approx 10^{-5}$. 
Figure 12. Comparison of the nonlinear model (thin solid lines) and linear model (thick solid lines) with experiment for the transmissibility of the pneumatic suspension: a) for $C_{r} \approx 10^{-8}$; (b) for $C_{r} \approx 10^{-7}$; (c) for $C_{r} \approx 10^{-6}$; (d) for $C_{r} \approx 10^{-5}$.

Figure 13. Transition frequency in the transmissibility results (linear model). 


\section{Tables}

Table 1. Comparison of the linear model and experimental results for the largest and the smallest values of the coefficient $C_{r}$ studied.

Table 1: Comparison of the linear model and experimental results for the largest and the smallest values of the coefficient $C_{r}$ studied.

\begin{tabular}{|c|c|c|c|c|c|}
\hline$C_{r}$ & Method & $f_{n}[\mathrm{~Hz}]$ & $\xi$ & $\%$ Deviation $f_{n}$ & \% Deviation $\xi$ \\
\hline$\sim 10^{-5}$ & Linear model & 2.26 & 0.102 & 2.5 & 7.2 \\
& Experimental & 2.32 & 0.110 & & \\
\hline$\sim 10^{-8}$ & Linear model & 3.054 & 0.075 & 1.0 & 11.7 \\
& Experimental & 3.088 & 0.085 & & \\
\hline
\end{tabular}


(a)

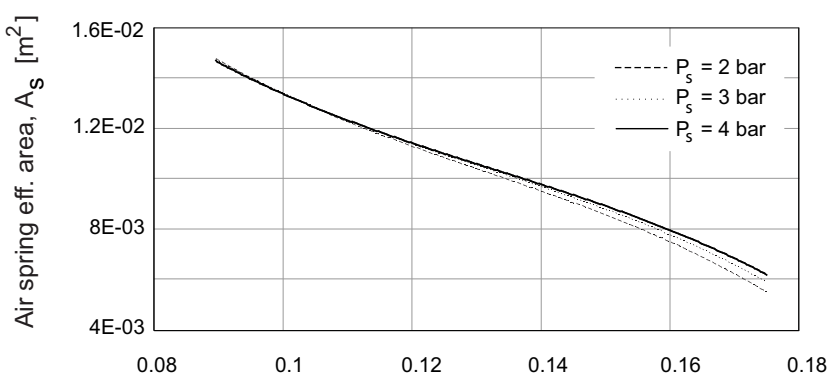

Height, z [m]

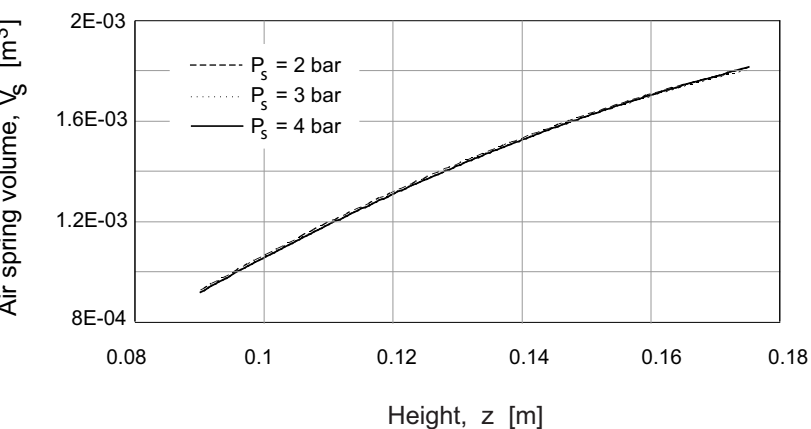

Figure 1: Experimental characterization of (a) the air spring effective area, and (b) the air spring volume.

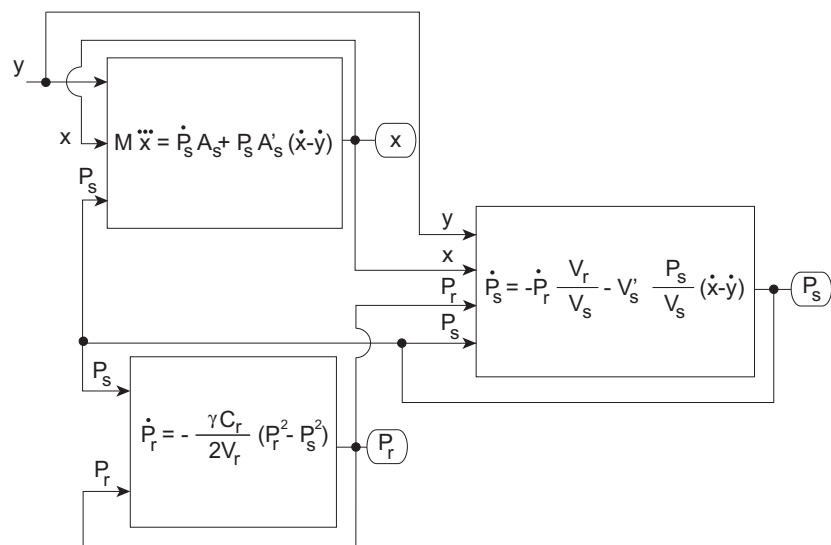

Figure 2: General Simulink block diagram for the nonlinear model of the pneumatic suspension. 
(a)

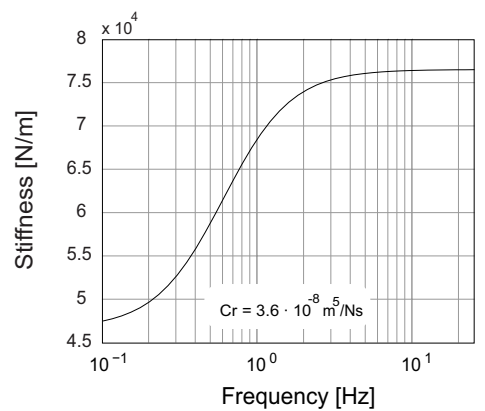

(b)

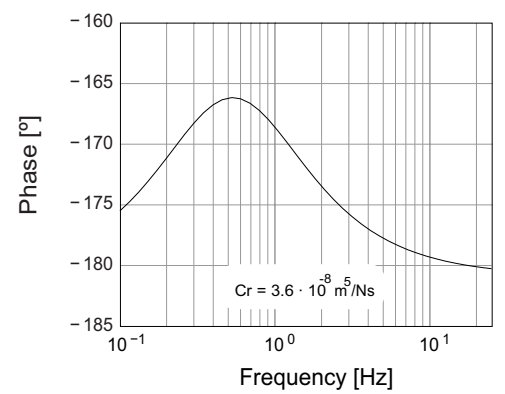

(e)

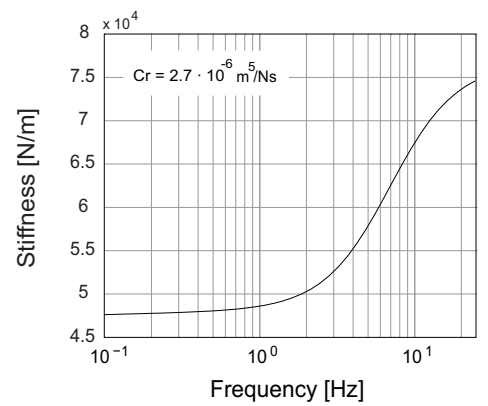

(f)

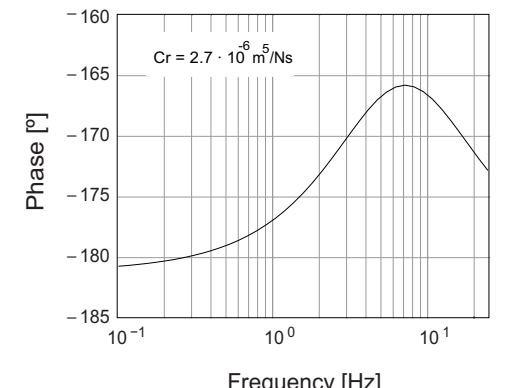

(c)

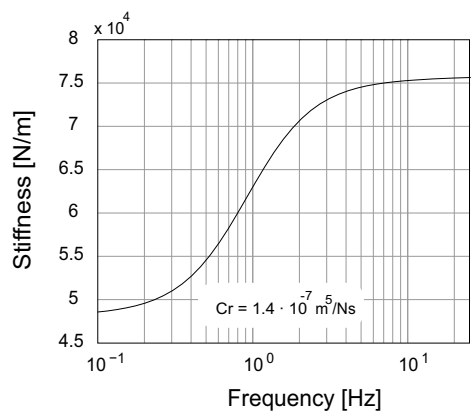

(d)

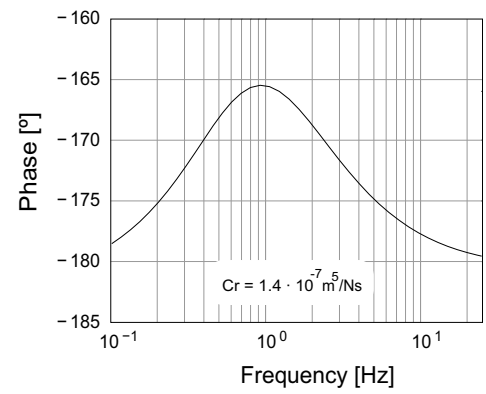

(g)

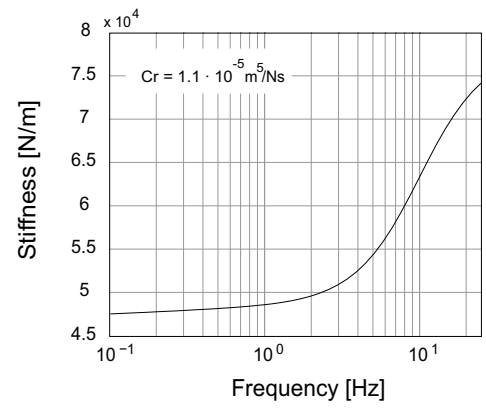

(h)

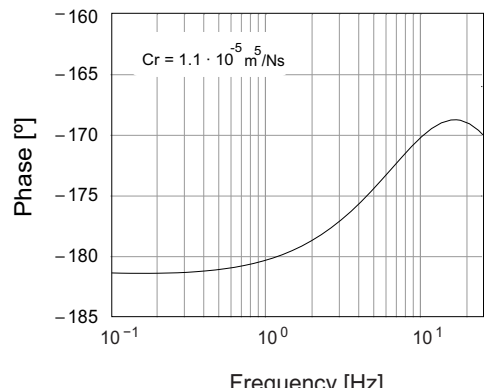

Figure 3: Nonlinear simulation (stiffness modulus and phase diagrams) for the suspension frequency response: (a) and (b) for $C_{r} \approx 10^{-8}$; (c) and (d) for $C_{r} \approx 10^{-7}$; (e) and (f) for $C_{r} \approx 10^{-6}$; (g) and (h) for $C_{r} \approx 10^{-5}$. 
(a)

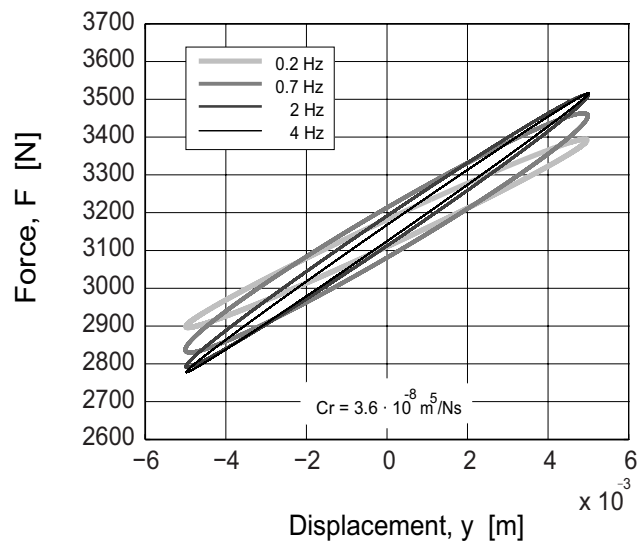

(c)

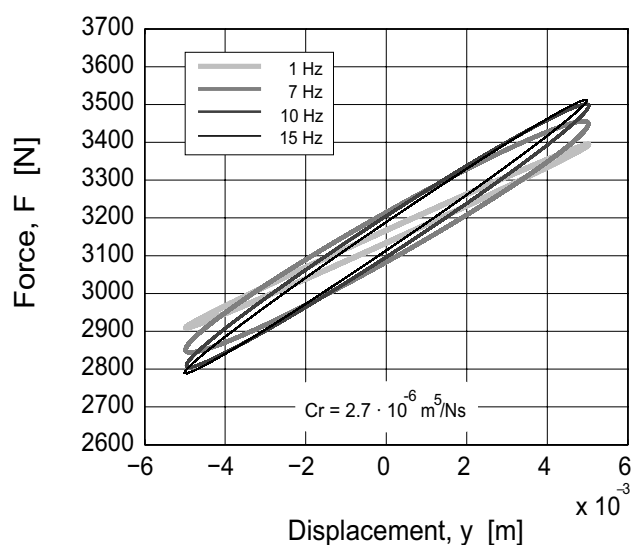

(b)

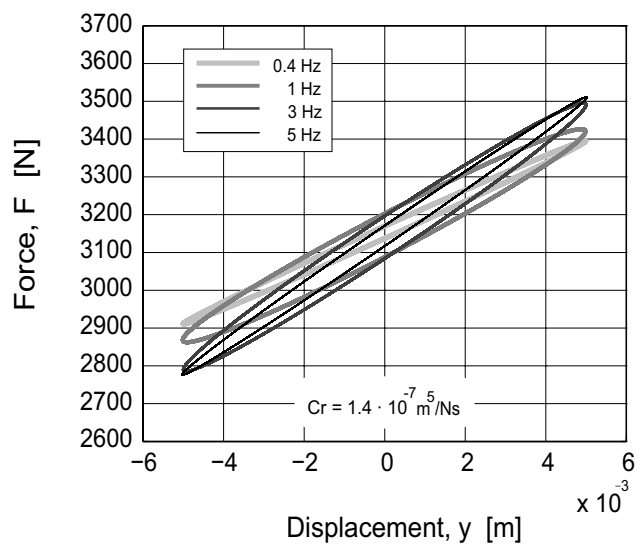

(d)

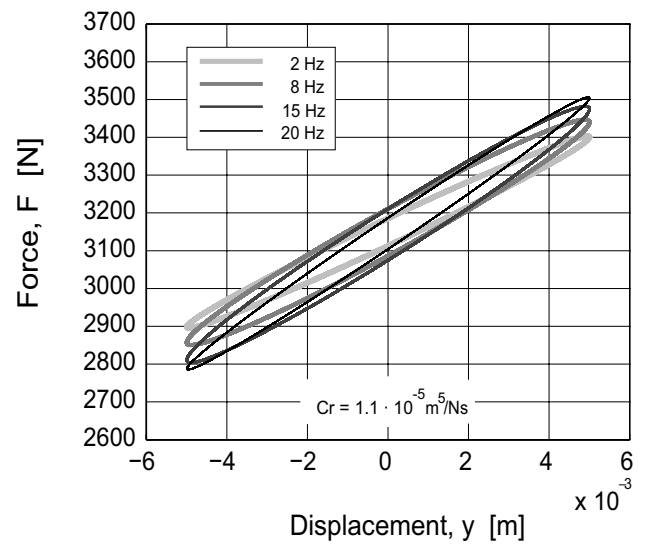

Figure 4: Nonlinear simulation for the suspension Carding diagram: a) for $C_{r} \approx 10^{-8}$; (b) for $C_{r} \approx 10^{-7} ;(\mathrm{c})$ for $C_{r} \approx 10^{-6} ;(\mathrm{d})$ for $C_{r} \approx 10^{-5}$.

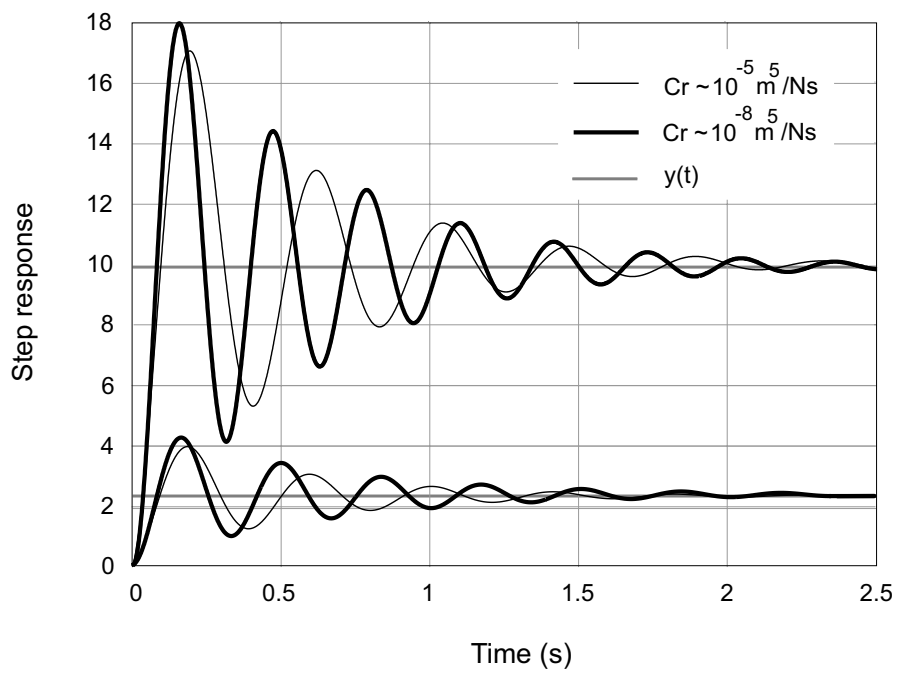

Figure 5: Nonlinear simulation for the suspension step response. 
(a)

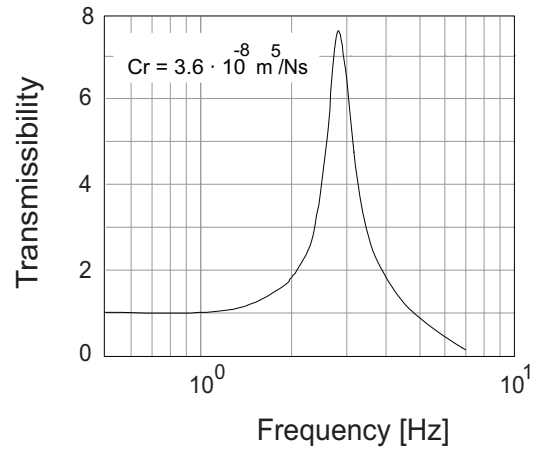

(c)

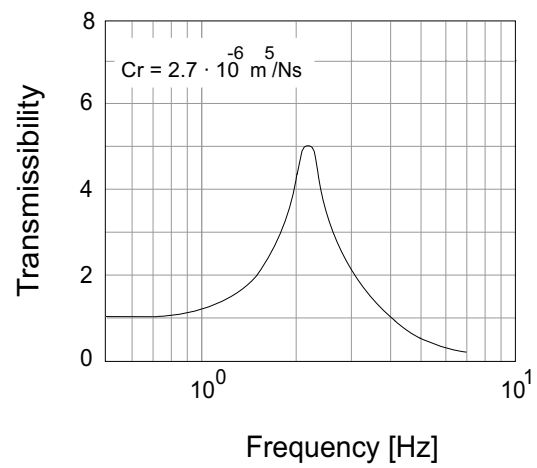

(b)

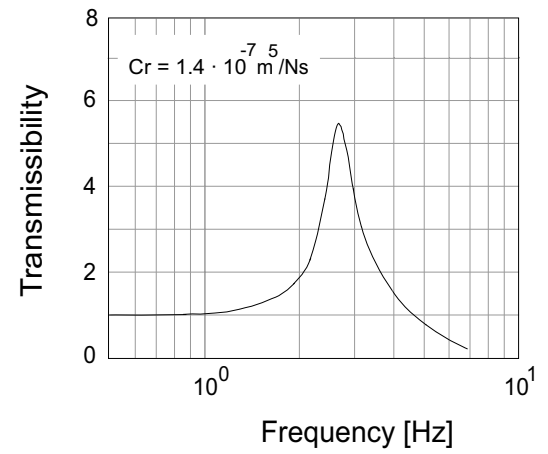

(d)

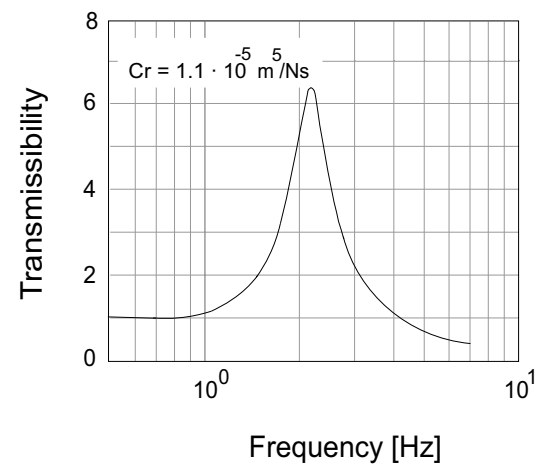

Figure 6: Nonlinear simulation for the suspension transmissibility: a) for $C_{r} \approx 10^{-8}$; (b) for $C_{r} \approx 10^{-7} ;(\mathrm{c})$ for $C_{r} \approx 10^{-6} ;$ (d) for $C_{r} \approx 10^{-5}$.
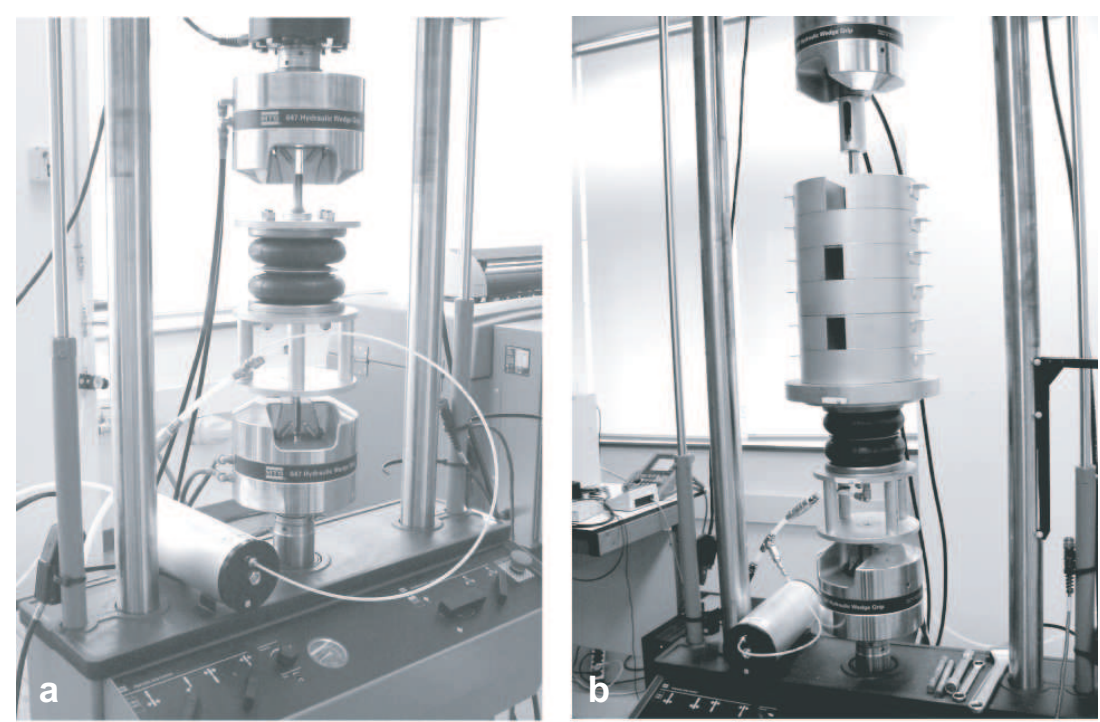

Figure 7: Experimental set-up: (a) stiffness measurements with no suspended mass; (b) damping and transmissibility measurements with suspended mass. 
(a)

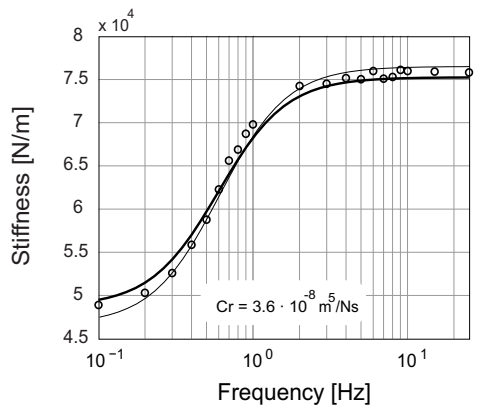

(b)

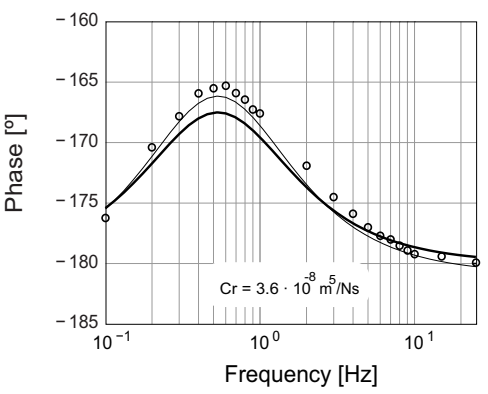

(e)

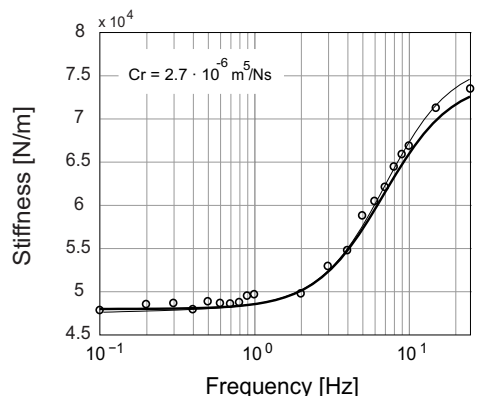

(f)

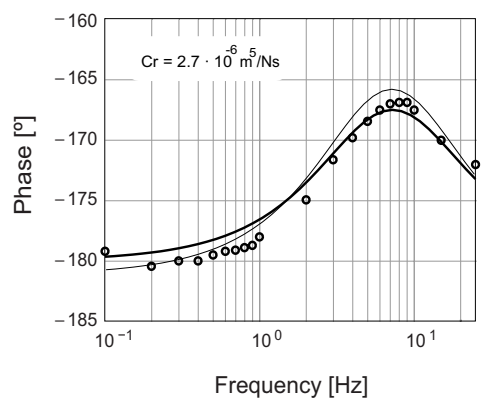

(c)

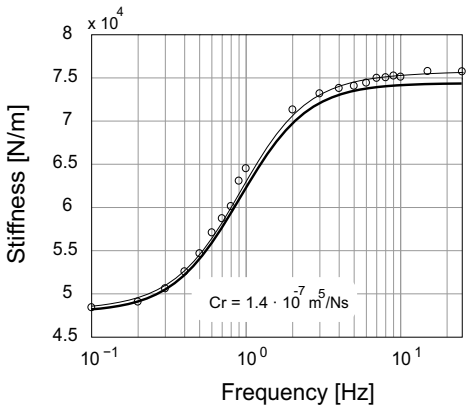

(d)

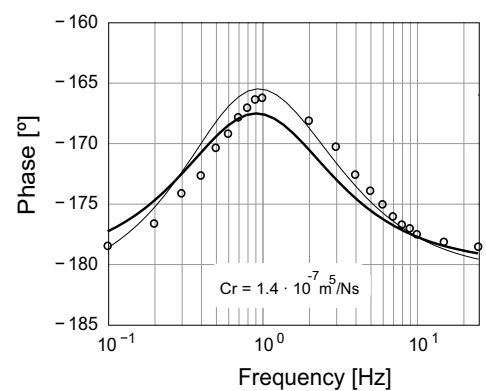

(g)

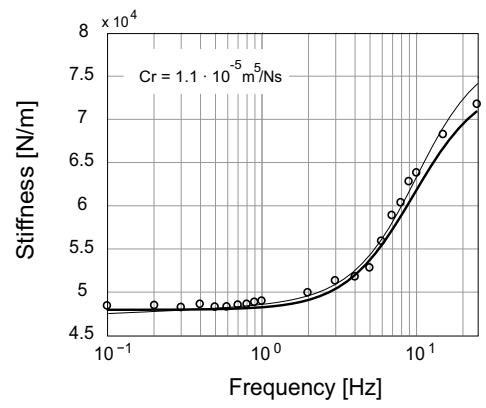

(h)

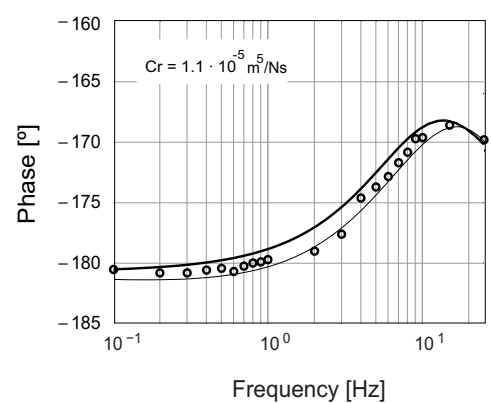

Figure 8: Comparison of the nonlinear model (thin solid lines) and linear model (thick solid lines) with experiment for the stiffness (modulus and phase diagrams) of the pneumatic suspension: (a) and (b) for $C_{r} \approx 10^{-8}$; (c) and (d) for $C_{r} \approx 10^{-7}$; (e) and (f) for $C_{r} \approx 10^{-6}$; (g) and (h) for $C_{r} \approx 10^{-5}$. 
(a)

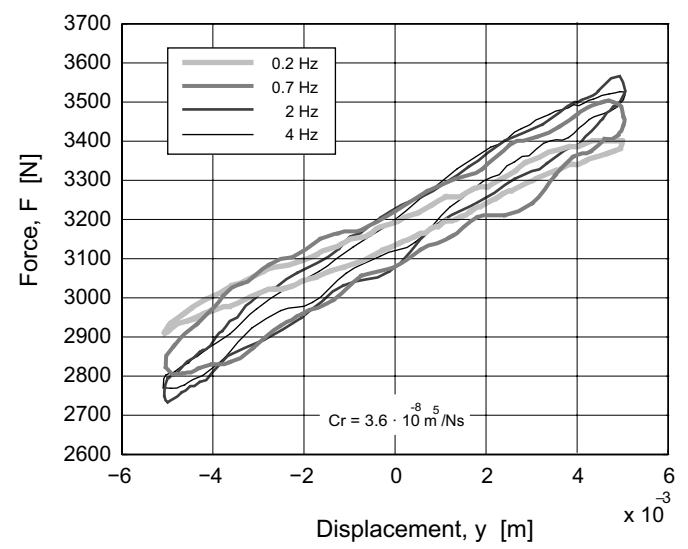

(c)

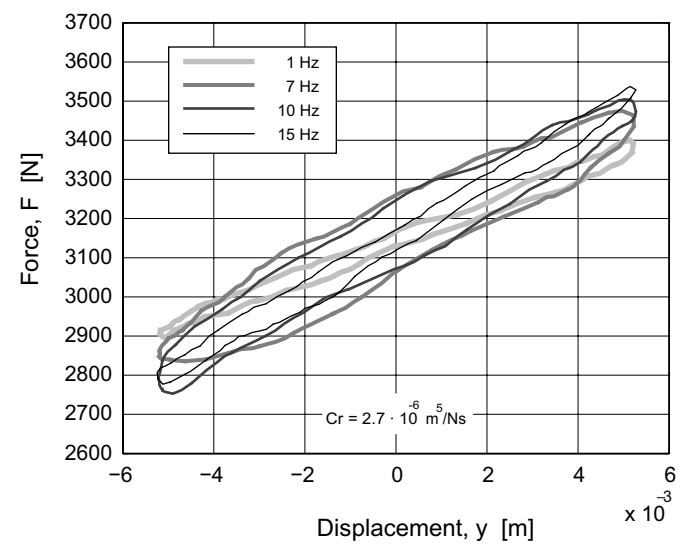

(b)

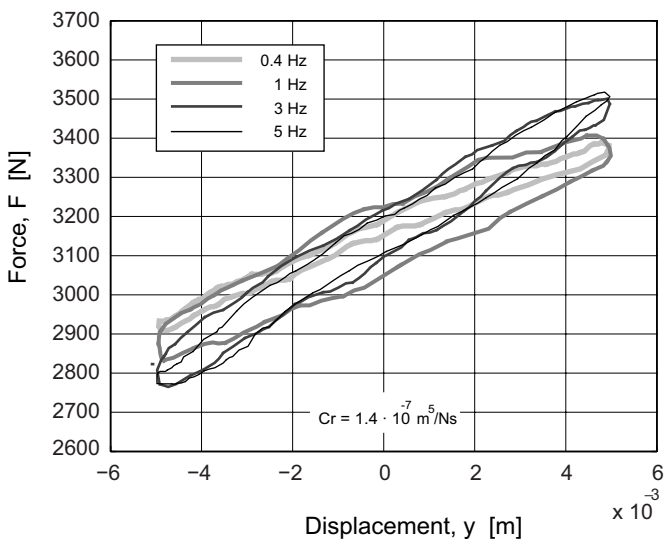

(d)

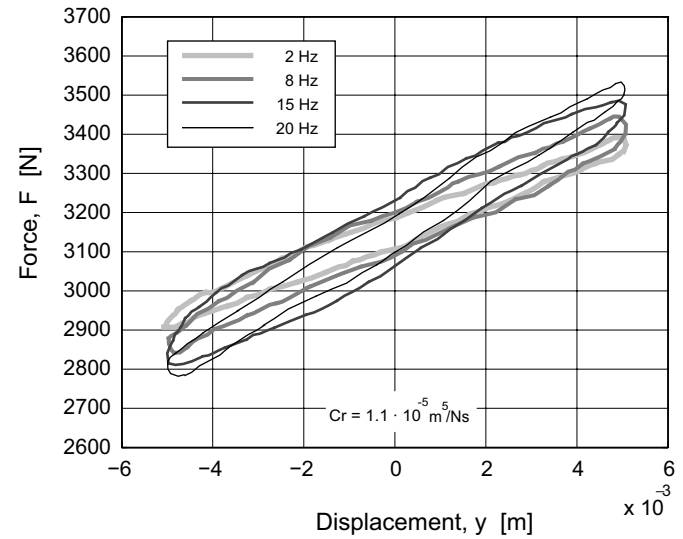

Figure 9: Test results for the suspension Carding diagram: a) for $C_{r} \approx 10^{-8}$; (b) for $C_{r} \approx 10^{-7}$; (c) for $C_{r} \approx 10^{-6}$; (d) for $C_{r} \approx 10^{-5}$.

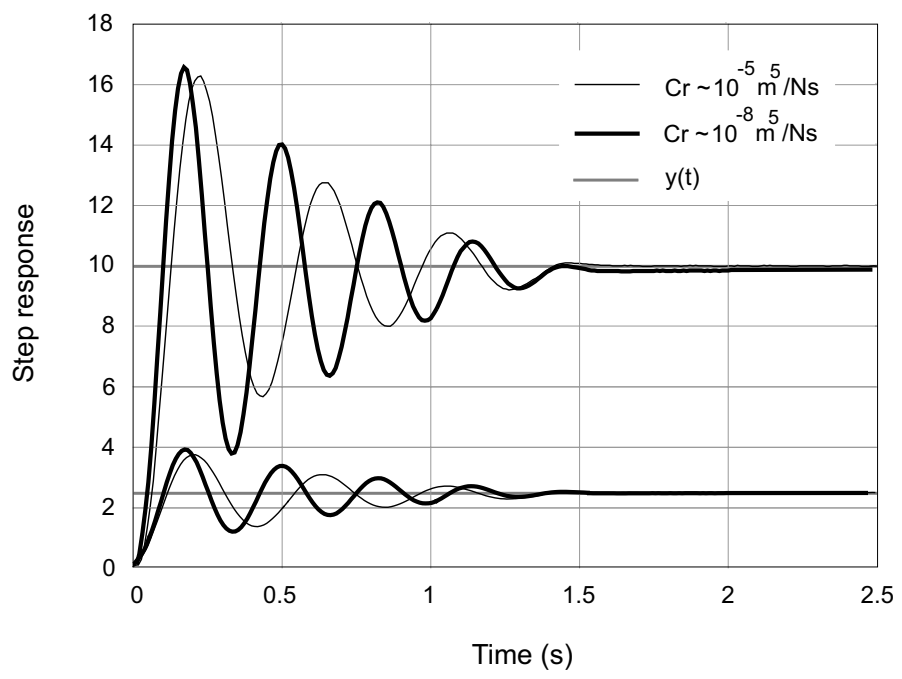

Figure 10: Test results for the suspension step response. 
(a)

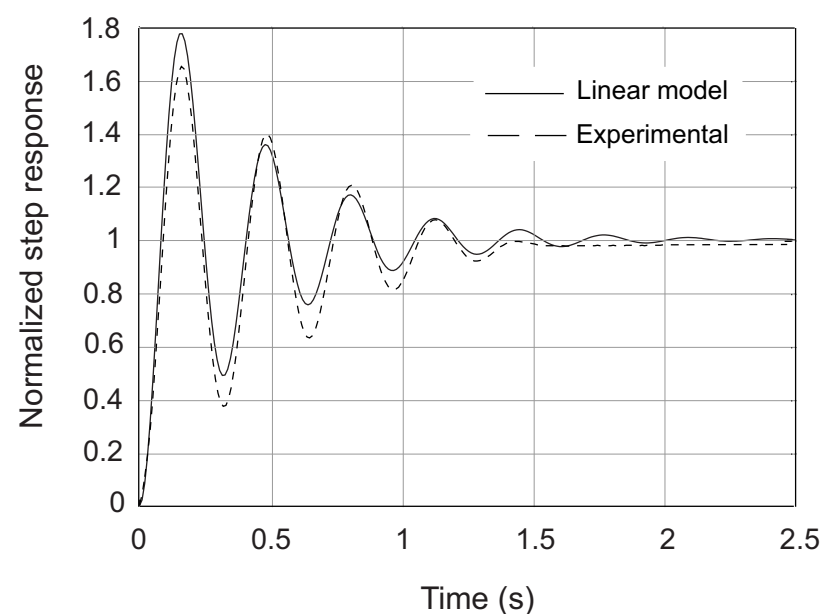

(b)

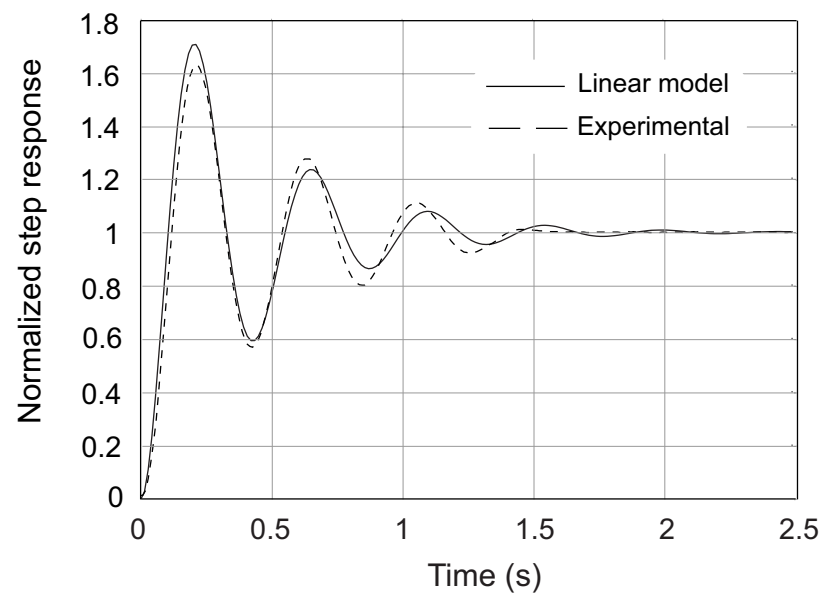

Figure 11: Comparison of the linear model (solid lines) with experiment for the suspension step response of the pneumatic suspension: (a) for $C_{r} \approx 10^{-8}$; (b) for $C_{r} \approx 10^{-5}$. 
(a)

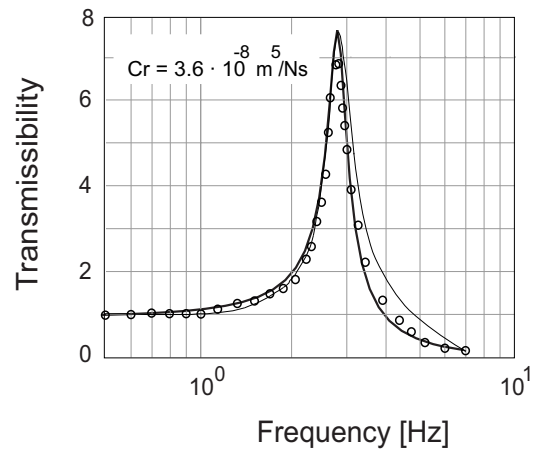

(c)

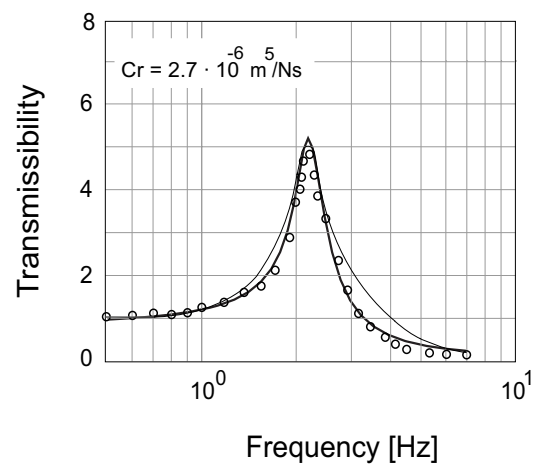

(b)

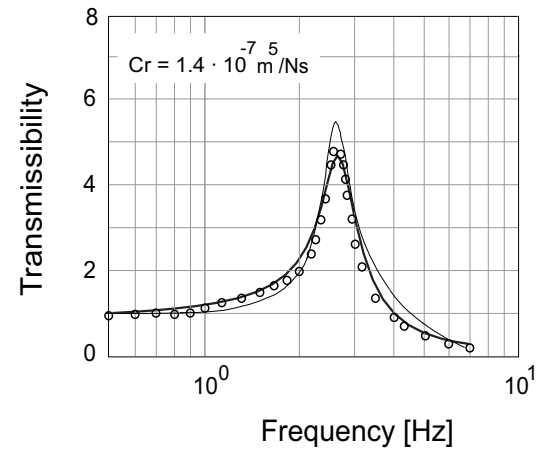

(d)

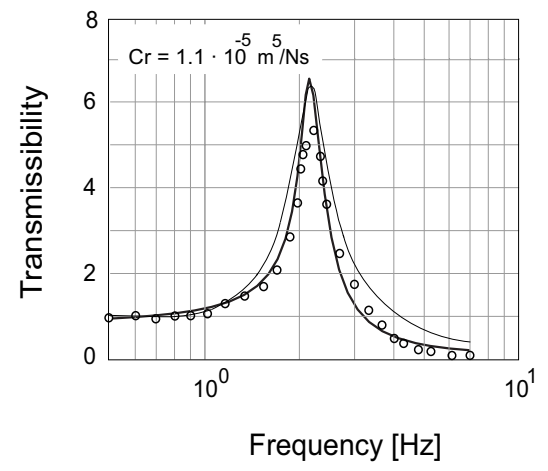

Figure 12: Comparison of the nonlinear model (thin solid lines) and linear model (thick solid lines) with experiment for the transmissibility of the pneumatic suspension: a) for $C_{r} \approx 10^{-8}$; (b) for $C_{r} \approx 10^{-7}$; (c) for $C_{r} \approx 10^{-6}$; (d) for $C_{r} \approx 10^{-5}$. 
(a)

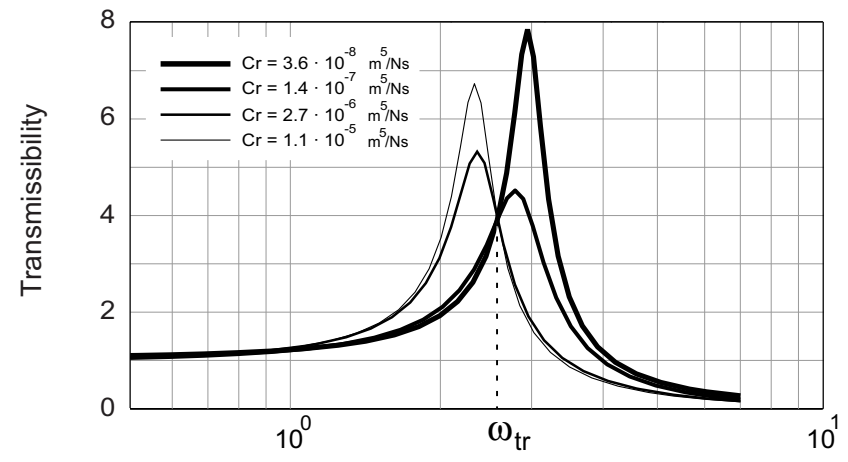

Frequency $[\mathrm{Hz}]$

(b)

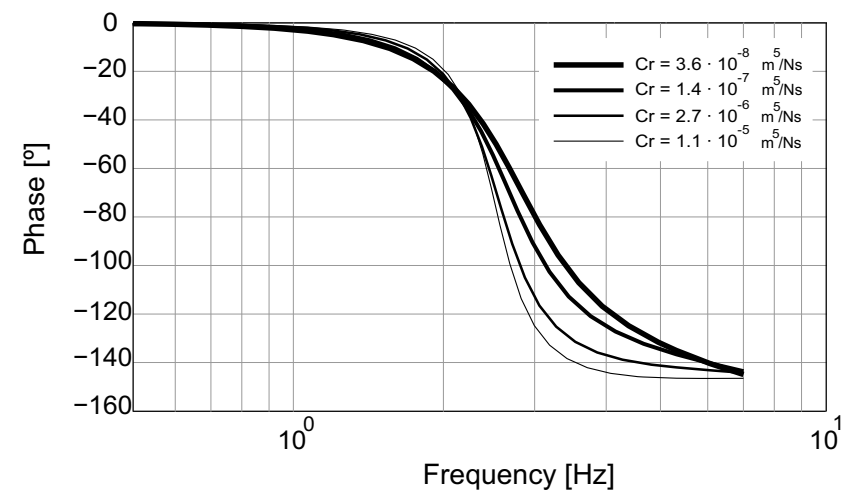

Figure 13: Transition frequency in the transmissibility results (linear model). 